\title{
Automated medical store management
}

\section{Dr. M.H.B. Ariyaratne}

Postgraduate Institute of Medicine, University of Colombo. Sri Lanka.

E-mail address: buddhika.ari@gmail.com

eHealth Sri Lanka 2010,1(suppl.1):S26

DOI: http://dx.doi.org/10.4038/sljbmi.v1i0.3571

Only the Abstract is available

\begin{abstract}
Efficiency and cost-effectiveness are essential components of a medical store management system. Manual systems lack several advantages of an automated computer system. A complete medical store management must have advanced features that lack in an ordinary stock management system. This paper will be useful to all who are still adhering to a manual system to think of implementing a computerised system, to others who are already using such a system to compare and add features as necessary, and to all who are involved in developing similar software. The auto order generation considers the trends of long term and short term past usage, usual monthly variations and the time taken by the particular supplier to deliver orders in the process of generating orders without any user intervention at the most appropriate time.

Though the process is automatic, it can be customised manually at any stage to meet any unpredictable variation. Reports like fast moving items, slow moving items, non moving will help increase the efficiency of the ordering process. The value of each item in the stock, especially in comparison to the value of utilisation will improve the cost effectiveness. In contrast to a normal stock management software, the stocks are handled batch vise. Different levels of warnings on impending drug expiry is another unique feature. As the items are categorised by generic name and the form, user can easily find possible alternatives during issuing a prescription and making orders. The ability to handle unlimited number of separate stores is essential when implementing in large scale institutions. The ability to inform the sub optimum availability of a certain item among different stores will prevent unnecessary ordering of an item when there is an excess in another unit.
\end{abstract}

Keywords - medical store management, stock management software 